\title{
Platelet lymphocyte ratio a diagnostic challenge in pancreatic head masses
}

\author{
Bollineny $\mathbf{V}^{1}$, Sateesh $\mathrm{S}^{2}$, Reddy $\mathbf{M}^{3}$, Konappa ${ }^{4}$, Reddy M.K \\ ${ }^{1}$ Dr Venkatesh Bollineny, Senior Resident, ${ }^{2}$ Dr Sreeram Sateesh, Professor \& Head, ${ }^{3}$ Dr Mahidhar Reddy, Associate \\ Professor, ${ }^{4}$ Dr Konappa, Assistant Professor, ${ }^{5}$ Dr Manoj Kumar Reddy, Assistant Professor, all authors are affiliated with \\ General Surgery, NMCH, Nellore, A.P, India.
}

Address for Correspondence: Dr Sreeram Sateesh, Professor \& Head, Department of General Surgery, NMCH, Nellore, A.P., Email: sreeramsateeshms@gmail.com

\begin{abstract}
Background: Masses in head of pancreas may arise from Carcinoma head of pancreas or Chronic pancreatitis. There is a need for differentiating the type of pancreatic head masses with utmost priority, since it can convert a radical surgery to conventional procedure. Recent studies suggest that Platelet lymphocyte ratio (PLR) can be used as a marker for differentiating between these masses. We evaluated the role of PLR and other tumour markers with combinations in differentiating the masses in head of the pancreas. Methods: A total of Sixty patients with mass in the head of pancreas with size $2 \mathrm{~cm}$ or greater were included in the study, with 30 patients in the Pancreatic ductal adenocarcinoma (PDA) group and 30 patients in the Chronic Pancreatitis group. Patients with evidence of acute pancreatitis, liver cirrhosis, cholangitis and obstructive jaundice, having Lewis antigen A/B blood types, with absent tissue/cytological diagnosis were excluded. Results: Haematological parameters like platelet count, neutrophil count, lymphocyte count were not statistically significanct between the two groups, whereas, Mean CA 19-9 levels, (PLR) Platelet lymphocyte ratio levels and (NLR) Neutrophil lymphocyte ratio in (PDA) Pancreatic ductal adenocarcinoma were higher than Chronic pancreatitis group. $(\mathrm{P}<0.05)$ In all these patients, biomarkers such as CA 19-9, Platelet lymphocyte ratio (PLR), Neutrophil lymphocyte ratio (NLR) and different combinations of these tumour markers were calculated, and their sensitivity, specificity,(PPV) Positive predictive value, (NPV) Negative predictive value and accuracy in differentiating malignant from benign pancreatic masses were established. The diagnostic accuracy of the tumour markers and their combinations are in the following order PLR $>$ PLR + CA 19-9 $>$ CA 19-9> CA 19-9+NLR> NLR. Conclusion: We found that PLR is as good as tumour marker CA 19-9 both individually as well as with other tumour marker combinations in the diagnosis of pathology of pancreatic head masses. Cost effectiveness, availability, simplicity and better diagnostic accuracy made PLR better tumour marker in the current scenario.
\end{abstract}

Keywords: Platelet lymphocyte ratio, Carcinoma head of pancreas, Chronic pancreatitis, Ca19-9

\section{Introduction}

Masses in the Head of the pancreas always pose a diagnostic dilemma to differentiate the nature, as they may arise from Carcinoma head of pancreas or Chronic pancreatitis. PDA (pancreatic ductal adenocarcinoma) develops in the head of the pancreas causing majority of cancer-related deaths owing to its aggressiveness. However, early diagnosis of such condition improves the survival rate. Some malignancies can also arise from the head of the pancreas secondary to chronic pancreatitis; it is wise to keep surveillance in patients with benign aetiology. Many Tumour markers like CA 19-9, tissue inhibitor of metalloproteinase-1 (TIMP-1), intercellular

Manuscript Received: $18^{\text {th }}$ June 2017

Reviewed: $28^{\text {th }}$ June 2017

Author Corrected: $7^{\text {th }}$ July 2017

Accepted for Publication: $13^{\text {th }}$ July 2017 adhesion molecule 1(ICAM 1) are available to diagnose the malignancy of pancreas [1-3]. Among them, CA 19-9, a sialylated Lewis blood group antigen has shown its consistency with well accepted sensitivity and specificity which varies with various cut-off values - $(<37 \mathrm{ku} / 1-81 \%$ \& 90\%), (100 ku/1-98\% \&68\%), (1000ku/1-99.8\% \& 41\%) [4]. However, CA 19-9 has got false negative results in conditions like Lewis blood type a, b and false positive results in obstructive jaundice and large tea consumers [57]. Goonnetilleke et al. stated that CA 19-9 should be used in the contemporary algorithm for the diagnosis of pancreatic malignancies [8]. The role of hematological parameters like Platelet lymphocyte ratio and Neutrophil lymphocyte ratio as tumor markers are subject of interest for diagnosis and prognostication [9, 10]. PDA causes 
thrombocytosis and leukocytopenia. Tumour produces cytokines (IL-6) which stimulate thrombocytosis as a part of paraneoplastic thrombocytosis. High platelets prevent tumour cells destruction from NK-cells and also helps in Tumour cells extravasation and angiogenesis. Clinically thrombocytosis may precede the diagnosis of PDA by months/years. Platelet lymphocyte ratio is also helpful in diagnosing the patients developing malignancy from an inflammatory setting, better than CA 19-9 [11].

R K. Miglani in a study stated that, PLR can be used for both diagnostic and prognostic purposes [12]. Smith et al [13] found that Pre-operative PLR is an independent predictor of survival on multivariate analysis. To this purpose, we evaluated the role of PLR and other tumour markers with combinations in differentiating the masses in head of the pancreas.

\section{Materials \& Methods}

This prospective study was conducted during May-2013 to April-2016 at Narayana medical college \& Hospital, Nellore.

Sample Size- A total of 60 patients with 30 patients in Pancreatic ductal adenocarcinoma group and 30 patients in Chronic pancreatitis group were included.

Inclusion Criteria- Sixty patients who were admitted with mass in the head of the pancreas with size $2 \mathrm{~cm}$ or greater were included.

Exclusion Criteria- Patients with evidence of acute pancreatitis, liver cirrhosis, cholangitis and obstructive jaundice, having Lewis antigen $\mathrm{A} / \mathrm{B}$, with absent tissue /cytological diagnosis were excluded.

Study Method- Demographic, personal and family histories were recorded from all the patients. Various risk factors for developing PDA and Chronic pancreatitis were gathered from the history such as family history with at least two affected first-degree relatives, environmental risk factors like Tobacco Chewing, Smoking and Alcoholism and medical risk factors like Pancreatitis, Diabetes ( $>$ 5years), and Obesity. Patients underwent CBP with PLR \& NLR, LFT, CA 19-9, USG Abdomen MDCT with Triphasic CT and CT-Angiography for assessment of resectability. PDA patients were grouped into resectable, borderline resectable and advanced disease.

Only $5 / 30$ patients were found out to be resectable and underwent Whipple procedure with R0 Resection. 3/30 patients were considered borderline resectable and within these patients, 2/30 patients had R1 Resection, and 1/30 had R0 Resection. 22/30 patients who had locally and distant metastasis, the diagnosis was made by intraoperative tissue biopsy while contemplating triple bypass and $\mathrm{CT}$ guided FNAC of metastasis.

In chronic pancreatitis, the majority of the patients without dilated main pancreatic duct underwent FREY procedure, and 4/30 patients with dilated MPD underwent BEGERS procedure. 6/30 patients who had adhesions due to recurrent pancreatitis underwent Pylorus-Preserving Pancreaticoduodenectomy.

Statistical Methods- Continuous variables were presented as the mean, median and standard deviation. Categorical variables were described in frequencies / percentiles. We used MaxStat statistical software for data processing and analysis. Comparison of the continuous variables of the study group was made using students' test and Mann-Whitney "U" test. Fisher's exact test was used to analyse absolute values.

Sensitivity, specificity, positive predictive value, negative predictive value and accuracy of each tumour markers and their combinations were calculated.Statistical significance was defined when $\mathrm{P}$ value $<0.05$.

\section{Results}

The two study groups contained 30 patients each with mass in the head of the pancreas due to PDA and chronic pancreatitis. When we analysed the differences in risk factors between these two groups, we found that smoking (40\%) and obesity (39\%) in PDA group and alcoholism (91\%) in chronic pancreatitis group were statistically significance. (Table-1). Nearly $60 \%$ of patients with PDA were above the age of 56 years, and $40 \%$ of patients were below 56 years. In $53 \%$ of patients, mass in the head of the pancreas due to chronic pancreatitis was noticed during their second and third decades of life.

Haematological parameters like platelet count, neutrophil count, lymphocyte count were not statistically significance between the two groups, whereas, Mean CA 19-9 levels, PLR levels and NLR in PDA were higher than Chronic pancreatitis group. $(\mathrm{P}<0.05)$ In all these patients, biomarkers such as CA 19-9, PLR, NLR and different combinations of these tumour markers were calculated, and their sensitivity, specificity, PPV, NPV and accuracy in differentiating malignant from benign pancreatic masses were established. The diagnostic accuracy of the tumour markers and their combinations are in the following order PLR $>$ PLR+CA 19-9> CA 19-9> CA 19-9+NLR > NLR (table-2) 
Original Research Article

Table-1: Demographic, clinical and haematological parameter comparison between the two disease groups.

\begin{tabular}{|c|c|c|c|}
\hline Clinical data & PDA & Chronic pancreatitis & $P$ value \\
\hline $10-20 \mathrm{Yrs}$ & 0 & $1(3.33 \%)$ & \multirow[t]{6}{*}{$<0.05$} \\
\hline $21-30 \mathrm{Yrs}$ & $1(3.33 \%)$ & $4(13.32 \%)$ & \\
\hline $31-40$ Yrs & $2(6.67 \%)$ & $8(26.64 \%)$ & \\
\hline 41-50 Yrs & $7(23.31 \%)$ & $8(26.64 \%)$ & \\
\hline 51-60 Yrs & $8(26.64 \%)$ & $6(19.98 \%)$ & \\
\hline$>60 \mathrm{Yrs}$ & $12(39.96 \%)$ & $3(9.99 \%)$ & \\
\hline Male : Female Ratio & $21: 9(2.3: 1)$ & $24: 6(4: 1)$ & $>0.05$ \\
\hline Family history & $4(12 \%)$ & $2(6 \%)$ & $>0.05$ \\
\hline Smoking & $12(40 \%)$ & $5(15 \%)$ & $<0.05$ \\
\hline Tobacco chewing & $2(6 \%)$ & $4(12 \%)$ & $>0.05$ \\
\hline Alcoholism & $7(21 \%)$ & $27(91 \%)$ & $>0.05$ \\
\hline Obesity & $13(39 \%)$ & $10(30 \%)$ & $<0.05$ \\
\hline Type-2 DM(>5years) & $9(27 \%)$ & $11(33 \%)$ & $>0.05$ \\
\hline Mean platelet count & $2.93 \pm 1.15$ & $2.42 \pm 1.21$ & $>0.05$ \\
\hline Mean lymphocyte count & $1601 \pm 330$ & $2028 \pm 407$ & $>0.05$ \\
\hline Mean Neutrophil count & $2587 \pm 225$ & $2370 \pm 384$ & $>0.05$ \\
\hline CA19-9 & $589 \pm 499$ & $102 \pm 101$ & $<0.05$ \\
\hline PLR & $227 \pm 128$ & $121 \pm 97$ & $<0.05$ \\
\hline NLR & $3.65 \pm 1.39$ & $1.25 \pm 1.17$ & $<0.05$ \\
\hline
\end{tabular}

Table- 2: Sensitivity, specificity, positive predictive value, negative predictive value and accuracy of each tumour markers and their combinations

\begin{tabular}{|c|c|c|c|c|c|}
\hline & Sensitivity & Specificity & PPV & NPV & Accuracy \\
\hline \multicolumn{6}{|l|}{ CA 19-9 } \\
\hline$>37 \mathrm{u} / 1$ & 91 & 50 & 69 & 92 & 68 \\
\hline$>100 \mathrm{u} / 1$ & 80 & 69 & 96 & 79 & 69 \\
\hline$>1000 \mathrm{u} / 1$ & 74 & 97 & 99 & 76 & 73 \\
\hline \multicolumn{6}{|c|}{ Platelet Lymphocyte ratio } \\
\hline$<150$ & 78 & 86 & 78 & 77 & 65 \\
\hline$>150$ & 71 & 90 & 80 & 64 & 70 \\
\hline \multicolumn{6}{|c|}{ Neutrophil Lymphocyte Ratio } \\
\hline$<2.5$ & 67 & 70 & 66 & 70 & 56 \\
\hline$>2.5$ & 60 & 72 & 69 & 64 & 66 \\
\hline \multicolumn{6}{|c|}{ Combinations of tumour markers } \\
\hline CA19-9+PLR & 72 & 88 & 74 & 67 & 68 \\
\hline CA 19-+NLR & 60 & 72 & 68 & 63 & 56 \\
\hline NLR $>2.5$ & 42 & 61 & 55 & 49 & 43 \\
\hline
\end{tabular}

\section{Discussion}

Pancreatic head masses always impose a diagnostic conundrum to the clinician. The obvious quagmire is to differentiate malignant and benign head masses.

Tumour markers are quite essential in early diagnosis, assessing the severity, prognosis and surveillance of the disease [14]. (ASCO) American Society of Clinical Oncology in its 2006 update concluded that CA 19-9 alone with its sensitivity and specificity is not adequate for diagnosis of pancreatic cancer [15]. National Academy of Clinical Biochemistry (NACB) suggested that CA19-9 should be combined with imaging modalities to increase the efficacy and strongly recommended studies for newer tumour markers to identify high-risk individuals, and with better sensitivity and specificity.

Tumour markers like CA19-9, PLR \& NLR and combinations were taken into this predicament, and PLR 
Original Research Article

showed superlative efficacy. Leukocytopenia is due to release of inflammatory cytokines (IL-1, IL-6 \&TNFalpha) which augment the cancer cells to metastasize by affecting the CRI (cancer related inflammation) cascade relating Lymphocytopenia with tumour aggressiveness /metastasis [9-11]

Smith et al., found that high pre-op PLR levels in suspected periampullary carcinoma with extensive tumour invasiveness and concluded that $1 / 5^{\text {th }}$ of laparoscopic surgeries could be avoidable if the combination of these markers is considered [16]. In cases of resected PDA, preop PLR was found to be more important tumour marker than other haematological parameters. The pre-op PLR found to be significant in multivariate analysis $(p<0.001)$ along with tumour size $(\mathrm{p}<0.001)$ and lymph node ratio $(\mathrm{p}<0.013)[16]$.

Rk Miglani et al. showed that- PLR can be used for both diagnostic and prognostic values and can differentiate malignant masses from benign aetiology. When used in combination the accuracy had increased [12].

In another study conducted by Bhatti et al., NLR retained significance in resected PDA than PLR [17]. In a study, the borderline resectable PDA patients were grouped based on pre-op PLR \& NLR for survival analysis and found that the NLR $>3$ and $P L R>225$ were independent prognostic factors in Borderline resectable PDA patients.

In our study, we have taken consideration of tumour markers like CA 19-9, PLR, NLR and their combinations. Many studies had proved CA19-9 as a diagnostic and prognostic tumour marker, but the level of acceptance by the clinician varies as the accuracy varied by various cutoff values. $\operatorname{PLR}(>150)$ was found to be a single independent prognostic factor on multivariate analysis with $(p<0.005)$ and the accuracy of the various combinations are PLR $>$ PLR + CA 19-9 $>$ CA 19-9 $>$ CA 19-9+NLR $>$ NLR.PLR was supportive in case of malignancy arising from chronic pancreatitis in 3 patients, however, is insignificant ( $p>0.005)$.PLR alone was found to be simple cost-effective and accurate diagnostic tumour marker than other tumour markers and combinations which yielded poor results.

\section{Conclusion}

We found that PLR is as good as tumour CA 19-9 both individually as well as with other tumour marker combinations in the diagnosis of pathology of pancreatic head masses. Cost effectiveness, availability, simplicity and better diagnostic accuracy made PLR better tumour marker in the current scenario.
Conflict of interest: None declared. Funding: Nil, Permission from IRB: Yes

\section{References}

1. Lee KJ, Yi SW, Chung MJ, Park SW, Song SY, Chung JB, Park JY. Serum CA 19-9 and CEA levels as a prognostic factor in pancreatic adenocarcinoma. Yonsei Med J. 2013 May 1;54(3):643-9. doi: 10.3349/ymj.2013. 54.3 .643 .

2. Tempero MA, Uchida E, Takasaki H, Burnett DA, Steplewski Z, Pour PM. Relationship of carbohydrate antigen 19-9 and Lewis antigens in pancreatic cancer. Cancer Res. 1987 Oct 15;47(20):5501-3.

3. Wu E, Zhou S, Bhat K, Ma Q. CA 19-9 and pancreatic cancer. Clin Adv Hematol Oncol. 2013 Jan; 11 (1): 53-5.

4. Bhat K, Wang F, Ma Q, Li Q, Mallik S, Hsieh TC, Wu E. Advances in biomarker research for pancreatic cancer. Curr Pharm Des. 2012;18(17):2439-51.

5. Duraker N, Hot S, Polat Y, Höbek A, Gençler N, Urhan N. CEA, CA 19-9, and CA 125 in the differential diagnosis of benign and malignant pancreatic diseases with or without jaundice. J Surg Oncol. 2007 Feb 1;95(2): $142-7$.

6. Howaizi M, Abboura M, Krespine C, Sbai-Idrissi MS, Marty O, Djabbari-Sobhani M. A new cause for CA19.9 elevation: heavy tea consumption. Gut. 2003 Jun;52 (6): 913-4.

7. Ørntoft TF, Vestergaard EM, Holmes E, Jakobsen JS, Grunnet N, Mortensen M, et al. Influence of Lewis $\alpha 1$ 3/4-L-fucosyltransferase (FUT3) gene mutations on enzyme activity, erythrocyte phenotyping, and circulating tumor marker sialyl-Lewis a levels. Journal of Biological Chemistry. 1996;271(50): 32260-8.http://www.jbc.org/ content /271/50/32260.long

8. Goonetilleke KS, Siriwardena AK. Systematic review of carbohydrate antigen (CA 19-9) as a biochemical marker in the diagnosis of pancreatic cancer. Eur J Surg Oncol. 2007 Apr;33(3):266-70. Epub 2006 Nov 9.

9. Kaser A, Brandacher G, Steurer W, Kaser S, Offner FA, Zoller H, Theurl I, Widder W, Molnar C, Ludwiczek O, Atkins MB, Mier JW, Tilg H. Interleukin-6 stimulates thrombopoiesis through thrombopoietin: role in inflammatory thrombocytosis. Blood. 2001 Nov 1;98(9): 2720-5. 


\section{Original Research Article}

10. Roxburgh CS, McMillan DC. Role of systemic inflammatory response in predicting survival in patients with primary operable cancer. Future Oncol. 2010 Jan; 6 (1): 149-63. doi: 10.2217/fon.09.136.

11. Palumbo JS, Talmage KE, Massari JV, La Jeunesse CM, Flick MJ, Kombrinck KW, Jirousková M, Degen JL. Platelets and fibrin(ogen) increase metastatic potential by impeding natural killer cell-mediated elimination of tumor cells. Blood. 2005 Jan 1;105(1):178-85. Epub 2004 Sep 14.

12. Miglani RK, Bhateja N, Bhat RS, Kumar KV. Diagnostic Role of Platelet Lymphocyte Ratio(PLR) in Pancreatic Head Masses. Indian J Surg. 2013 Feb;75(1): 4-9. doi: 10. 1007/s12262-012-0443-6. Epub 2012 Mar 27.

13. Smith RA, Bosonnet L, Raraty M, Sutton R, Neoptolemos JP, Campbell F, et al. Preoperative plateletlymphocyte ratio is an independent significant prognostic marker in resected pancreatic ductal adenocarcinoma. The American Journal of Surgery. 2009;197(4):466-72. https: // www. ncbi.nlm.nih.gov/pubmed/18639229.
14. Bhat K, Wang F, Ma Q, Li Q, Mallik S, Hsieh TC, $\mathrm{Wu}$ E. Advances in biomarker research for pancreatic cancer. Curr Pharm Des. 2012;18(17):2439-51.

15. Locker GY, Hamilton S, Harris J, Jessup JM, Kemeny N, Macdonald JS, Somerfield MR, Hayes DF, Bast RC Jr; ASCO. ASCO 2006 update of recommendations for the use of tumor markers in gastrointestinal cancer. J Clin Oncol. 2006 Nov 20;24(33):5313-27. Epub 2006 Oct 23.

16. Smith RA, Bosonnet L, Ghaneh P, Sutton R, Evans J, Healey $\mathrm{P}$, et al. The platelet-lymphocyte ratio improves the predictive value of serum CA19-9 levels in determining patient selection for staging laparoscopy in suspected periampullary cancer. Surgery. 2008 May;143 (5):658-66. PubMed PMID: 18436014. Epub 2008/04/26. eng. https://www.ncbi.nlm.nih. gov/pubmed / 18436014

17. Bhatti I, Peacock O, Lloyd G, Larvin M, Hall RI. Preoperative hematologic markers as independent predictors of prognosis in resected pancreatic ductal adenocarcinoma: neutrophil-lymphocyte versus plateletlymphocyte ratio. Am J Surg. 2010 Aug;200(2):197-203. doi: 10.1016/j.amjsurg.2009.08.041. Epub 2010 Feb 1.

\section{How to cite this article?}

Bollineny V, Sateesh S, Reddy M, Konappa, Reddy M.K. Platelet lymphocyte ratio a diagnostic challenge in pancreatic head masses.Int J surg Orthopedics.2017;3(3):55-59.doi:10.17511/ijoso.2017.i03.01. 